\title{
Capital Humano e Crescimento Econômico: Uma análise dos Municípios de Pernambuco entre 2000-2010
}

\author{
Álvaro Robério de Souza SÁ \\ Universidade Federal Rural de Pernambuco (UFRPE), Serra Talhada, PE, Brasil \\ Dhiego Lúcio da SILVA \\ Universidade Federal Rural de Pernambuco (UFRPE), Serra Talhada, PE, Brasil \\ Maria Solange Nunes de Lima SÁ \\ Universidade Federal Rural de Pernambuco (UFRPE), Serra Talhada, PE, Brasi
}

Received 1 Out. 19; Accepted 17 Out. 19

Evaluation System: Double Blind Review

Editor: Jose Lindenberg Julião Xavier Filho, Dr.

ISSN: $2594-8040$

To cite this paper: Sá, A. R. S., SILVA, D. L., \& Sá, M. S. N. (2019). Capital Humano e Crescimento Econômico: Uma análise dos Municípios de Pernambuco entre 2000-2010. Journal of Perspectives in Management - JPM, 3(2), p. 35-48.

\section{Resumo}

A educação se apresenta como um fator primordial para o crescimento e desenvolvimento econômico. Nesse contexto, este estudo mensurou a contribuição da taxa de alfabetização sobre o crescimento da renda per capita dos municípios de Pernambuco, entre 2000 e 2010, através do arcabouço teórico de Solow (1956) e Mankiw et al. (1992). Para alcançar esse objetivo, utilizou-se um painel de dados balanceado, com as variáveis extraídas de fontes oficiais, a citar IBGE, BDE e IPEADATA. Os resultados evidenciaram que o aumento de $1 \%$ no consumo de energia (capital físico) e taxa de alfabetização (capital humano) provocam uma variação positiva de $0,19 \%$ e $0,38 \%$ na renda per capita dos municípios do Estado de Pernambuco. Constatou-se, ainda, que em todos os modelos estimados os efeitos do investimento em capital humano são superiores aos do capital físico sobre a renda per capita.

Palavras-Chave: Crescimento Econômico, Capital Humano, Educação, Pernambuco. 


\section{Introdução}

O estado de Pernambuco iniciou um processo de grandes transformações econômicas no início do século XXI, mesclando uma política de investimento em infraestrutura produtiva e formação de capital humano. Essa mudança de preferências tem promovido o crescimento econômico, através dos avanços educacionais, que impactam positivamente na renda per capita das economias municipais.

A evolução do Índice de Desenvolvimento Humano Municipal (IDHM) de Educação, entre 2000 e 2010, demonstra a ascensão do capital humano, como fator de produção relevante na determinação do progresso econômico. Nesse período, a economia pernambucana obteve um crescimento de 29,7\% no IDHM-Educação, saltando do patamar de 0,538 para 0,698. No mesmo intervalo, o Produto Interno Bruto (PIB) do estado cresceu a uma taxa média anual de 4,2\%, superior à média da economia nacional, que ficou em 3,7\% ao ano.

No ano de 2000, o investimento bruto do estado de Pernambuco na educação correspondia a 1,53\% do Produto Interno Bruto (PIB), enquanto em 2010 esse montante subiu para 2,42\%, representando um crescimento de $57,8 \%$ na razão educação/produto. Nesse período, ainda, os dispêndios médios da educação pernambucana alcançaram o patamar de $\mathrm{R} \$ 1,129$ bilhão, refletindo um aumento de $469 \%$ na década (Secretaria do Tesouro Nacional, 2011). Esse aumento no investimento com educação associado as políticas educacionais de nível nacional e estadual, adotadas entre 2000 e 2010, corroboraram para melhoria dos indicadores de qualidade educacional do estado.

Segundo o Atlas de Desenvolvimento Humano no Brasil (2012), no ano 2000, 66,6\% da população de 6 a17 anos de Pernambuco estavam cursando o ensino básico regular com até dois anos de defasagem idade/série. Após uma década, esse percentual saltou para o patamar de 79,24\%, representando uma evolução de $18,9 \%$. No ano de 2000 , os pernambucanos estudavam em média 7,7 anos; após uma década, esse número passou para 9,13 anos. Nesse contexto, a taxa de analfabetismo dos indivíduos por faixa etária de idade, respectivamente, era de 13,4\% (11 a 14 anos), $10,8 \%$ (15 a 17 anos), 13,2\% (18 a 24 anos) e 29,7\% (maiores de 25 anos), reduziram para $5,6 \%, 4,2 \%, 5,7 \%$ e $22,2 \%$, respectivamente.

Esses números refletem uma melhora significativa no quadro da educação do estado de Pernambuco, gerando um importante efeito na capacidade de produção da economia, tendo em vista que a escolarização aumenta a produtividade marginal dos trabalhadores e, por conseguinte, promove o crescimento econômico.

Diante desse cenário, o estudo do capital humano tem sido cada vez mais relevante à explicação da evolução dos níveis de renda das economias. Essa importância tem sido constatada através dos efeitos diretos e indiretos causados pelo avanço da educação sobre a produção agregada. Os efeitos da escolarização da força de trabalho incidem, principalmente, no desenvolvimento de tecnologias, que são incorporados ao processo produtivo, promovendo o aumento da produção e, consequentemente, o progresso econômico (Cangussu et al., 2010).

O impacto do capital humano sobre o crescimento econômico tem como precursores Mincer (1958), Schultz (1961) e Becker (1964). Posteriormente, Lucas (1988), Romer (1990) e Mankiw et al. (1992) incorporam esse fator de produção ao modelo de Solow (1956), com intuito de explicar o crescimento econômico.

Assim, este estudo tem como finalidade mensurar a contribuição da taxa de alfabetização para o crescimento econômico dos municípios de Pernambuco, entre 2000 e 2010. Especificamente, tem-se o intuito de verificar como a evolução da educação na década impacta no Produto Interno Bruto (PIB) per capita, através do modelo de Solow ampliado. 
Nesse sentindo, este trabalho contribui com os estudos sobre o crescimento econômico ao dar ênfase a importância da alfabetização da população, como fonte de ampliação da renda per capita dos municípios pernambucanos. $\mathrm{O}$ estudo, ainda, reforça a importância do capital humano como um dos determinantes da evolução econômica. $\mathrm{O}$ artigo apresenta, além dessa breve introdução; seção (2), fundamentação teórica; seção (3) metodologia; seção (4), resultados e discussões e seção (5) considerações finais.

\section{Fundamentação Teórica}

\subsection{Teoria do Capital Humano}

A importância da educação no crescimento econômico consolidou-se com a teoria do capital humano ao enfatizar que os investimentos na qualificação profissional do trabalhador ampliariam sua capacidade produtiva e, consequentemente, promoveriam o progresso da economia.

A priori, Mincer (1958) apontou a existência de uma correlação entre investimento na formação profissional e distribuição de renda pessoal. Nesse sentindo, a escolha individual e racional do trabalhador na alocação do tempo em capacitação, treinamento e aquisição de novos conhecimentos definiria o seu nível de capital humano e os seus rendimentos pessoais. Portanto, quanto mais o indivíduo investe em educação maiores retornos adquire e mais produtivo torna-se, impactando, assim, positivamente no progresso da economia.

Por outro lado, Schultz (1961) enfatizou que os dispêndios com educação são heterogêneos entre os indivíduos dados os diferentes rendimentos. Portanto, as qualificações adquiridas pelo trabalhador dependem da relação entre ganhos adicionais e custos para adquiri-las. Para este autor, a escolarização da população, além de ampliar a produtividade da força de trabalho também promoveria o bem-estar social, sobretudo dos trabalhadores mais pobres. Nesse contexto, a qualificação e o aperfeiçoamento profissional da população, provenientes dos aportes em educação, elevariam a produtividade da força de trabalho e expandiriam os lucros das firmas.

Para Becker (1964), o trabalhador adquire capacidades produtivas através da acumulação de capital humano geral e específico. Deste modo, a escolha do indivíduo em obter mais habilidades profissionais parte de um trade-off entre deixar de receber renda no presente para obter maiores rendimentos no futuro, isto é, aplicar recursos e tempo em educação no presente para obter maiores retornos posteriormente. Esse é o custo de oportunidade que o trabalhador se defronta ao decidir alocar seu tempo entre trabalhar com baixo estoque de capital humano e receber rendimentos menores, ou dedicar-se aos estudos e ter a possibilidade de auferir salários maiores no futuro. No entanto, destaca-se que a produtividade do trabalhador não depende apenas da sua aptidão e dos dispêndios em educação, outros fatores são importantes também, tais como motivação e qualidade do ambiente de trabalho.

No que tange a acumulação de capital humano, o esforço do indivíduo em obter treinamento e qualificação depende do conjunto de suas características pessoais e intrínsecas aos estudos, ou seja, cada trabalhador absorve a educação de maneira diferenciada, sendo esse um dos motivos para explicar os diferentes níveis de produtividade dos trabalhadores.

Nesse panorama, quando o estoque de capital humano aumenta, em virtude da ampliação dos investimentos na estrutura educacional, os retornos do estoque de capital crescem até encontrar um equilíbrio, isto é, até a renda per capita aumentar dado a relação direta entre educação e crescimento econômico (Becker et. al, 1990).

Destaca-se, ainda, o efeito positivo e repercutido da acumulação de capital humano pelos indivíduos, que promovem um processo de escolarização intergeracional entre os agentes econômicos, impactando positivamente ao longo do tempo na economia. Por essa razão, cada vez mais as economias têm investido em educação e formação profissional, afim de obter retornos positivos do estoque de capital da economia.

Pode-se concluir, então, que os efeitos diretos e indiretos do capital humano no crescimento da renda per capita incidem, principalmente, sobre o progresso tecnológico (Nakabashi e Figueirêdo, 2005). Portanto, o capital humano é um insumo fundamental no processo de criação, 
aquisição e difusão da tecnologia no médio e longo prazo, sendo este também um dos determinantes do crescimento das economias, como será apresentado através das contribuições de Romer (1990).

\subsection{Teoria Neoclássica do Crescimento Econômico}

A teoria neoclássica emerge com uma importante contribuição de Robert Solow $(1956 ; 1957)$ ao desenvolvimento econômico. Esse teórico desenvolveu um modelo de crescimento que permite estimar a renda per capita das economias, a partir de uma função de produção do tipo CobbDouglas com retornos constantes de escala para descrever o aumento da acumulação dos fatores de produção, respectivamente, capital físico $(K)$ e trabalho $(L)$, multiplicados por uma escalar tecnológica $(A)$. A função pode ser expressa da seguinte forma:

$Y(t)=K^{\propto}(A L)^{1-\infty}, 0<\alpha<1$

No modelo tem-se que $\propto$ e $(1-\infty)$ são elasticidades do produto $(Y)$ em relação aos insumos de produção, enquanto a escalar tecnológica é exógena as escolhas dos agentes econômicos. Portanto, choques exógenos de progresso tecnológico expandem a configuração dos fatores da produção agregada no estado estacionário.

Para Abramovitz (1986), o progresso técnico, também chamado de resíduo de Solow, corresponde a maior parte da explicação do crescimento das economias, tendo em vista que um conjunto de fatores não incorporados no modelo de crescimento são também relevantes para descrever o progresso econômico.

O modelo de Solow foi aperfeiçoado por outros teóricos neoclássicos através da exploração do resíduo. Nesse sentindo, emerge a corrente teórica do crescimento econômico endógeno, que passam a incorporar novas variáveis que também são responsáveis por determinar o crescimento das economias.

Essa nova vertente parte do trabalho seminal de Lucas (1988) sobre o capital humano ao introduzir as escolhas racionais dos agentes econômicos, visando aperfeiçoar a teoria do crescimento neoclássico, haja vista ser esse um fator dinâmico nas economias. Nessa perspectiva, Romer (1990) propõe o primeiro modelo de crescimento econômico com progresso tecnológico, a partir das decisões dos agentes econômicos privados, frente aos incentivos de mercado para ampliação da produtividade. Logo, ao incorporar o capital humano, este pressupõe que o progresso tecnológico se trata de um componente endógeno. Portanto, o modelo de crescimento com capital humano explora o resíduo de Solow, ampliando a capacidade de explicação do progresso econômico, através da ampliação da função de produção neoclássica.

Deste modo, o capital humano passa a ser considerado como o motor do crescimento econômico, partindo-se da dinâmica de que o trabalhador empreende seu tempo em educação, capitação profissional e ampliação das suas habilidades técnicas, formando o estoque de capital humano da economia (Lucas, 1988). Assim, a função de acumulação de capital humano é expressa por:

$\dot{h}(t)=h(t)^{\xi} G[1-u(t)]$

Onde $h(\mathrm{t})$ representa a taxa acumulação de capital humano no período $t$, sendo determinado por $h(t)$, o nível já alcançado de capital humano, e por $G[1-u(t)]$, a dedicação dos agentes a obtenção de mais qualificação $t$ écnica (educação). Nesse sentindo, a dedicação dos indivíduos racionais é composta de um trade-off entre lazer $u(t)$ e esforço para absorção de mais conhecimento, qualificação e capacitação. Dessa maneira, quanto menor for o tempo dedicado ao lazer $u(t)$ maior será acumulação de capital humano da economia $h(t)$. 
Tanto o efeito interno do capital humano através da habilidade individual $h$ quanto pelo efeito externo, por meio da elevação do nível de capital humano $h_{a}$, afetam a produtividade dos demais fatores de produção da economia. Assim, substituindo $h$ e $h_{a}$ na função de produção de Solow obtém-se a função de produção com capital humano de Lucas (1988), que pode ser descrita por:

$Y(t)=A K(t)^{\beta}[u(t) N(t) h(t)]^{1-\beta} h_{a}(t)^{\mu}$

Tem-se, então, que $h_{a}(t)^{\mu}$ representa a captura das externalidades do capital humano, enquanto $[1-u(h)]$ corresponde a dedicação, acumulação de capital humano. Assim, considera-se que o progresso tecnológico esteja incorporado ao crescimento do estoque de capital humano $h(t)$, que por sua vez é determinado em função de $h(t)$ e do tempo de dedicação a absorção de novas habilidades profissionais.

Por essa razão, a acumulação de capital humano ao nível individual $h$ produz uma externalidade positiva a nível global. Assim, o conhecimento de um indivíduo permite que os outros se beneficiem desse conhecimento. A partir da função de produção de Lucas (1988) é possível definir que quanto mais elevado for o nível médio de capital humano $h_{a}$, maior será a produtividade dos fatores influenciados por externalidades geradas por $h_{a}$ e captadas por $\mu$. Portanto, considerando que o progresso tecnológico esteja incorporado em $h$ e $h_{a}$, tem-se o aumento do produto e, consequentemente, o crescimento econômico.

Dando continuidade à teoria do crescimento econômico endógena, Romer (1990) parte das premissas de que o progresso tecnológico é o motor do crescimento econômico e incentivador da acumulação continuada do capital; o progresso técnico resulta da ação dos agentes racionais que respondem positivamente a incentivos do mercado (tornando o modelo endógeno) e, por fim, considera que a tecnologia é um bem não rival e parcialmente excludente.

Esses pilares direcionam Romer (1990) a considerar que o estoque de capital humano de uma economia é alocado para a produção de bens manufaturados, através da força de trabalho ou no desenvolvimento de inovações, por meio de pesquisa e desenvolvimento (P\&D).

Assim, somente economias que distribuem o estoque de trabalho para esses enfoques são capazes de inovar e monopolizar os lucros gerados pela criação de novas ideias, processos ou tecnologias, temporariamente, isto é, até que ocorra a difusão tecnológica. Nesse sentindo, as mudanças técnicas devem ser compreendidas como quaisquer transformações no processo produtivo que gerem ganhos de eficiência e produtividade, através da melhor combinação de insumos no processo de criação de novos bens para o mercado capitalista (Romer, 1990).

Desse modo, o capital humano pode ser definido como o acúmulo de conhecimentos proporcionados pelos anos de educação formal e capacitação profissional dos trabalhadores, sendo representados da seguinte forma:

$Y\left(H_{y^{\prime}}, L, x\right)=H_{y}^{\alpha} L^{\beta} \int_{0}^{\infty} x_{(i)}^{1-\infty-\beta} d i$

Onde $x(i)$ indica o i-ésimo insumo de capital físico, enquanto $L$ e $H_{y}$ são os estoques de trabalho e capital humano presentes na economia. Nesse sentido, a produção não é afetada de forma direta pelo progresso tecnológico, mas indiretamente por meio da inovação tecnológica que propicia a criação de um novo bem durável a ser incorporado no processo de produção, capaz de gerar um novo bem final ou quando o progresso tecnológico promove a elevação da produtividade dos fatores de produção (Salgueiro, 2012). Portanto, o crescimento econômico originário do progresso tecnológico pode ser determinado pela quantidade de trabalho alocado em atividades de pesquisa e desenvolvimento $(\mathrm{P} \& \mathrm{D})$, onde o capital humano representa o determinante do progresso tecnológico e inovação da economia. 


\subsection{Modelo de Solow ampliado com Capital Humano}

O modelo de crescimento econômico de Mankiw et al. (1992) parte das contribuições de Lucas (1988) e Romer (1990), a partir de uma função de produção neoclássica com a integração do capital humano como fator de produção da economia. Desse modo, o produto agregado da economia pode ser expresso por:

$Y=K^{\alpha} H^{\beta}(A L)^{1-\alpha-\beta}$

Onde o produto agregado $(Y)$; capital físico $(K)$; capital humano $(H)$; trabalho $(L)$ e tecnologia $(A)$, enquanto $\alpha, \beta$ e $(1-\alpha-\beta)$ correspondem a participação dos fatores de produção em relação ao produto da economia.

Segundo Mankiw et al. (1992), tem-se que a tecnologia é poupadora de trabalho. Assim, o termo $(A L)$ corresponde ao fator por unidade efetiva de trabalho que cresce a uma taxa de $(n+g)$, onde $n$ taxa de crescimento populacional e $g$ taxa de progresso tecnológico, a seguir:

$L(t)=L(0) e^{n t}$

$A(t)=A(0) e^{g t}$

Desse modo, os fatores da função de produção (5) podem ser definidos em forma de fatores em unidades efetivas de trabalho: $\hat{y}=\frac{Y}{A L} ; \hat{k}=\frac{K}{A L} ; \hat{h}=\frac{H}{A L}$. Logo, considerando que $K$ e $H$ se depreciam a uma taxa $\delta$, as equações que expressam as acumulações de capital físico e capital humano podem ser expressas por:

$\dot{k}=s_{k} y-(n+g+\delta) k$

$\dot{h}=s_{h} y-(n+g+\delta) h$

Onde $s_{k}$ corresponde a fração da renda investida em capital e $s_{h}$ fração da renda investida na acumulação de capital humano. Assim, igualando as equações (6.1) e (6.2) a zero, obtêm-se $k$ e $h$ no estado estacionário:

$k^{*}=\left[\begin{array}{l}\frac{s_{k}^{1-\beta} s_{h}^{\beta}}{n+g+\delta} \\ \frac{\beta}{(1-\alpha-\beta)}\end{array}\right]^{\frac{1}{(\alpha-\beta)}}$
$h^{*}=\left[\frac{s_{k}^{\alpha} s_{h}^{1-\alpha}}{n+g+\delta}\right]^{\frac{1}{(1-\alpha-\beta)}}$

Tomando as equações de estado estacionário do capital físico e do capital humano, respectivamente, (7.1) e (7.2), e substituindo na função de produção apresentada em unidades efetivas de trabalho $\hat{y}=\widehat{k}^{\propto} \widehat{h}^{\beta}$ encontra-se a renda per capita do estado estacionário:

$y^{*}=\left[\frac{1}{n+g+\delta}\right]^{\frac{\alpha+\beta}{1-\alpha-\beta}}\left[s_{k}^{\alpha} s_{h}^{\beta}\right]^{\frac{1}{1-\alpha-\beta}}$

Colocando a escalar tecnológica $(A)$ em evidência e aplicando logaritmo em ambos os lados da equação (8), encontra-se o produto per capita da economia no estado estacionário:

$\ln y^{*}=\ln A_{0}+g t-\left(\frac{\alpha+\beta}{1-\alpha-\beta}\right) \ln (n+g+\delta)+\left(\frac{\sigma}{1-\approx-\beta}\right) \ln \left(s_{k}\right)+\left(\frac{\beta}{1-\alpha-\beta}\right) \ln \left(s_{k}\right)$ 
Onde gt é a taxa de crescimento do progresso tecnológico. O termo $A_{0}$ representa os fatores que estão implícitos no modelo (fatores geográficos, institucionais, dentre outros externos). Sendo assim, esse termo pode variar entre as economias, Mankiw et al. (1992) assume que:

$\ln A_{t}=a+\varepsilon$

Em que $a$ é uma constante e $\varepsilon$ representa uma especificada de cada economia. A equação acima será utilizada para a estimação das produtividades municipais. Substituindo (10) em (9), encontra-se

$\ln y^{*}=a+g t+\left(\frac{\approx}{1-\alpha-\beta}\right) \ln \left(s_{k}\right)+\left(\frac{\beta}{1-\alpha-\beta}\right) \ln \left(s_{k}\right)-\left(\frac{\pi+\beta}{1-\alpha-\beta}\right) \ln (n+g+\delta)+\varepsilon$

A equação (11) representa a variação da renda per capita no estado estacionário e será utilizada para as regressões nesse trabalho. Segundo Mankiw et al. (1992) essa equação com inclusão do capital humano responde por cerca de $80 \%$ da variação da renda per capita entre as economias. Esses autores, ainda, evidenciaram que o capital humano é um fator de produção de grande relevância para explicar o diferencial de renda e crescimento das diferentes economias.

\section{Procedimentos Metodológicos}

Para estimar o efeito do capital físico e capital humano sobre a renda per capita dos municípios de Pernambuco, entre 2000 e 2010, foi utilizado um modelo empírico em logaritmo natural $(l n)$, cuja estimação determina os parâmetros de elasticidades.

O período de 2000 e 2010 foi escolhido para evitar ruídos nas estimações, tendo em vista que diversos problemas econômicos ocorreram nesse intervalo, dentre eles: choques adversos, crise energética, crise externa, taxa de câmbio depreciando e crise financeira global. No entanto, o principal motivo trata dos avanços econômicos e sociais que o estado de Pernambuco alcançou durante essa primeira década do século XXI.

Tendo em vista a natureza longitudinal dos dados da pesquisa de municípios $(i)$ no tempo $(t)$, serão utilizados os estimadores dos dados em painel com efeito fixo e aleatório. Para identificar o estimador mais consistente serão realizados os testes de Chow, Breuch-Pagan e Hausman. Os referidos testes apontarão se o melhor modelo de estimação é o Pooled, Efeito Fixo ou Efeito Aleatório.

\subsection{Modelo Empírico}

O modelo empírico é resultado do modelo de crescimento de Solow ampliado representado pela equação (11), que assume as seguintes especificações no modelo econométrico:

$$
\ln y_{i t}=\ln \beta_{\text {oit }}+\beta_{1} \ln s_{\text {kit }}+\beta_{2} \ln s_{\text {hit }}+\beta_{3} \ln \left(n_{i t}+g_{i t}+\delta_{i t}\right)+u_{i t}
$$

onde $y$ renda per capita; $s_{k}$ fração da renda investida em capital físico; $s_{h}$ fração da renda investida em capital humano; $n$ taxa de crescimento populacional, $g$ taxa de progresso tecnológico e $\delta$ taxa de depreciação, enquanto $i$ corresponde aos municípios, $t$ o tempo e $u$ distúrbio aleatório.

\subsection{Banco de Dados e Descrição das Variáveis}

A base de dados utilizada é formada por 184 municípios do estado de Pernambuco compreendendo o período de 2000 e 2010. Os dados foram obtidos através de fontes oficiais como Instituto Brasileiro de Geografia e Estatística (IBGE), Instituto de Pesquisa Econômica Aplicada (IPEADATA) e Banco de Dados do Estado (BDE) de Pernambuco. 
As variáveis utilizadas foram: 1) o Produto Interno Bruto (PIB) a preços correntes, deflacionado pelo deflator implícito (ano base 2000); 2) população residente, sendo que as taxas são estimativas das populações residentes nos municípios entre 1999-2000 e 2009-2010; 3) taxa de alfabetização (taxa de alfabetização=100-taxa de analfabetos) como proxie de capital humano; 4) consumo de energia em mega-watts-hora como proxie de capital físico; 6) progresso tecnológico e depreciação foram utilizados os valores assumidos por Mankiw et al. (1992) de 0.05 (Firme e Simão Filho, 2014).

Quadro 1: Descrição das variáveis do modelo econométrico

\begin{tabular}{lcc}
\hline Variável & Descrição & Fonte \\
\hline$y_{i t}$ & Produto Interno Bruto (PIB) a preços correntes & IBGE \\
$k_{\text {it }}$ & Consumo de Energia Elétrica & BDE \\
$h_{\text {it }}$ & Taxa de Alfabetização & BDE \\
$n_{\text {it }}$ & População residente & IPEADATA \\
$g_{i t}+\delta_{i t}$ & Taxa de Progresso Tecnológico e Depreciação (0.05) & Mankiw et al. (1992) \\
\hline
\end{tabular}

Fonte: Elaboração própria (2019).

\section{Resultados e Discussão}

A estimativa da equação do modelo de Solow ampliado pressupõe a consistência empírica das relações do estoque de capital físico e capital humano e produto per capita. No que tange a esse aspecto da análise, a Figura 01 deixa claro o ajuste das referidas proxies em relação ao produto per capita dos municípios do Estado de Pernambuco.

Figura 01: Nuvem de dispersão das relações entre o produto per capita e a formação do capital físico e humano dos municípios de Pernambuco 2000-2010
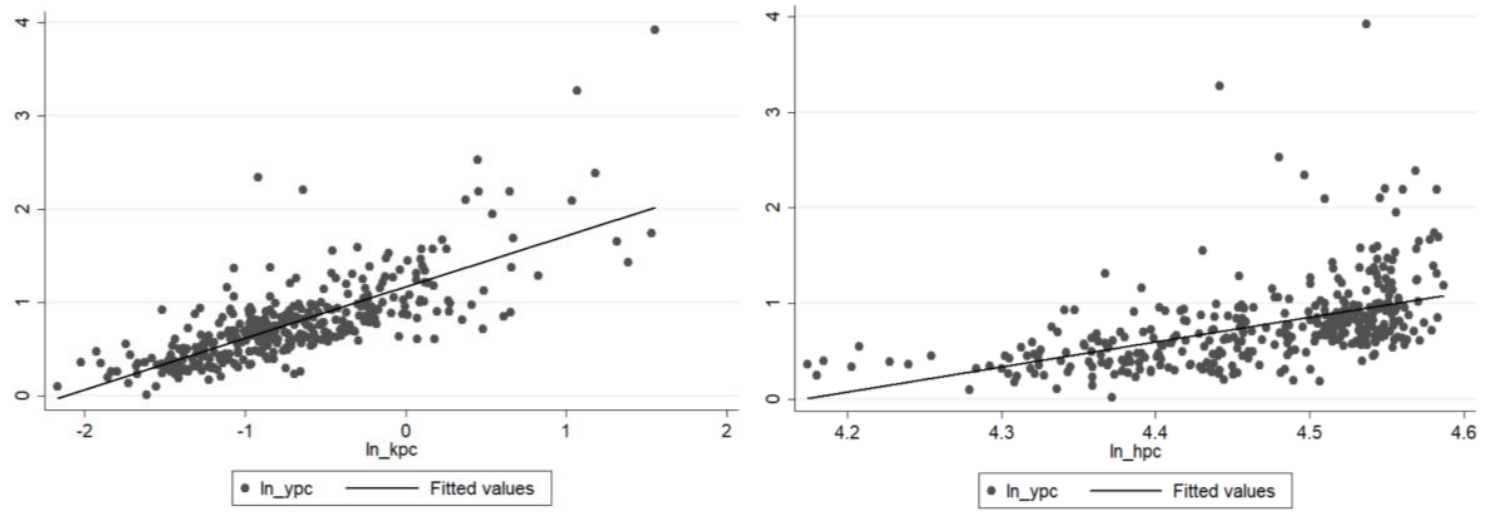

Fonte: Elaboração própria com base no resultado da pesquisa (2019).

As nuvens de dispersão do PIB per capita em relação a proxie de consumo de energia per capita (capital físico) e taxa de alfabetização (capital humano) mostrou-se positivamente relacionada e concentrada em torno da tendência, apesar do gráfico apresentar alguns outliers. Essas relações condizem com a teoria do crescimento econômico de Solow (1956) e Mankiw et al. (1992), ou seja, elevados estoques de capitais físico e humano propiciam um maior nível de produto per capita à economia.

Ilustram-se na Tabela 1 as estatísticas descritivas das variáveis utilizadas para estimar o modelo de Solow ampliado para os municípios do Estado de Pernambuco no período de 2000 e 2010. Abaixo apresenta-se a descrição do Produto Interno Bruto (PIB) a preços correntes, Consumo de Energia, Taxa de Alfabetização e População Residente.

A variável PIB a preços correntes $\left(y_{i t}\right)$ apresentou um valor médio por município de $\mathrm{R} \$$ 331.696,50 (em mil R\$); consumo de energia $\left(k_{i t}\right)$ valor médio de 47221.37 mega-watts-hora; taxa 
de alfabetização $\left(h_{i t}\right)$ média de $87,54 \%$ e população residente $\left(n_{i t}\right)$ média de 44.507 . Ao todo foram 368 observações para 184 municípios de Pernambuco.

Tabela 1: Estatística descritiva das variáveis utilizadas no modelo em análise

\begin{tabular}{ccccc}
\hline Variáveis & Média & Desvio-Padrão & Mínimo & Máximo \\
\hline$y_{\text {it }}$ & 331696.5 & 1801147.00 & 7226.00 & 30200000.00 \\
$k_{\text {it }}$ & 47221.37 & 210791.50 & 1129.00 & 2862418.00 \\
$h_{\text {it }}$ & 87.54049 & 7.133242 & 64.98 & 98.17 \\
$n_{\text {it }}$ & 44507.71 & 125280.40 & 2033.00 & 1500000.00 \\
\hline
\end{tabular}

Fonte: Elaboração própria (2019).

A Tabela 2 apresenta os resultados do modelo empírico proposto de acordo com os métodos de estimação com dados empilhados, com efeito fixo e com efeito aleatório, sem e com controle de tendência. Todas as estimativas foram realizadas com desvio-padrão robusto dos parâmetros, inibindo o efeito de problemas associados a heterocedasticidade e autocorrelação serial.

Em praticamente todos os casos os coeficientes exibem parâmetros estimados com o sinal esperado pela relação teórica, as únicas diferenças se dão em termos de desvio-padrão e significância estatística. Independente da especificação apresentada e do estimador, os resultados demonstram que existe um efeito positivo do capital físico e, especialmente, do capital humano sobre o produto per capita dos municípios do estado de Pernambuco entre 2000 e 2010.

A hipótese assumida de acordo com as descobertas de Mankiw et al. (1992) é de que a inclusão do fator capital humano na função de produção neoclássica explica a maior parte da variação na renda per capita das economias. Nesse sentindo, em quase todas as especificações, os fatores capitais físico e humano apresentaram significâncias estatísticas de 1\%,5\% e 10\%, porém, a depreciação não apresentou significância em nenhum dos modelos estimados. Os resultados são coerentes com os achados da teoria neoclássica sobre o capital humano.

Assim, as especificações que exibiram maior efeito causal do capital humano sobre a renda per capita foi o OLS e os estimadores de efeito fixo e aleatório sem tendência. Destaca-se que, cada modelo apresentou coeficientes, desvio-padrão e significância estatística diferentes. No entanto, o estimador de feito fixo com dummy de tempo se mostrou o mais coerente em relação a especificação assumida, devido a um conjunto de testes. Todos os testes de hipóteses conjunta de que os parâmetros são iguais a zero são rejeitados a $1 \%$ de significância, conforme as estatísticas dos testes F e Wald.

Entre as especificações com dados empilhados contra o efeito fixo com tendência, o teste de Chow rejeita a hipótese de que o estimador de OLS é preferível ao modelo com efeito fixo. O mesmo é observado para o teste do modelo OLS em relação ao estimador de efeito aleatório com tendência, através do teste de Breusch e Pagan. O estimador de OLS com dados empilhados se mostrou menos consistente do que o modelo com tendência. Entre os estimadores de efeito fixo e aleatório com tendência, o teste de Hausman rejeita a hipótese de que a diferença entre os coeficientes é sistemática. Dessa maneira, não é possível afirmar que o modelo de efeito aleatório com tendência seja mais consistente que o de efeito fixo com tendência. Logo, aceita-se a hipótese alternativa de que existem características não observáveis inerentes aos municípios do Estado de Pernambuco, invariantes no tempo, que afetam a produção agregada. Portanto, aceita-se o estimador de efeito fixo com tendência como o mais adequado.

O referido estimador demonstra que o parâmetro de capital físico é estatisticamente significativo a um nível de confiança de $99 \%$, enquanto o do capital humano é ao nível de confiança de $90 \%$. 
Tabela 2: Estimativas do modelo de Solow Ampliado (MRW) segundo o método de estimação com painel balanceado

\begin{tabular}{|c|c|c|c|c|c|c|}
\hline Variáveis & OLS & $\begin{array}{l}\text { OLS com } \\
\text { tendência }\end{array}$ & Efeito fixo & Efeito aleatório & $\begin{array}{c}\text { Efeito fixo com } \\
\text { tendência }\end{array}$ & $\begin{array}{l}\text { Efeito aleatório } \\
\text { com tendência }\end{array}$ \\
\hline $\operatorname{lnkpc}$ & $\begin{array}{l}0.4942651 * * * \\
(0.0604339)\end{array}$ & $\begin{array}{c}0.1997961 * * * \\
(0.0375743)\end{array}$ & $\begin{array}{l}0.2784057 * * * \\
(0.0433276)\end{array}$ & $\begin{array}{l}0.3952475^{* * *} \\
(0.0632789)\end{array}$ & $\begin{array}{l}0.1997961 * * * \\
(0.0373568)\end{array}$ & $\begin{array}{c}0.3665263 * * * \\
(0.0635285)\end{array}$ \\
\hline $\operatorname{lnh} p c$ & $\begin{array}{l}0.7025843 * * * \\
(0.2450304)\end{array}$ & $\begin{array}{l}0.3827364^{*} \\
(0.2033178)\end{array}$ & $\begin{array}{l}1.411634 * * * \\
(0.1591169)\end{array}$ & $\begin{array}{l}1.065155 * * * \\
(0.2000294)\end{array}$ & $\begin{array}{c}0.3827364^{*} \\
(0.202179)\end{array}$ & $\begin{array}{l}0.4269111^{*} \\
(0.2246598)\end{array}$ \\
\hline $\ln (n+g+d)$ & $\begin{array}{c}0.076875 \\
(0.0556801)\end{array}$ & $\begin{array}{r}-0.0068572 \\
(0.043314)\end{array}$ & $\begin{array}{l}-0.0282268 \\
(0.0448235)\end{array}$ & $\begin{array}{c}0.0105712 \\
(0.0471787)\end{array}$ & $\begin{array}{l}-0.0068572 \\
(0.0430714)\end{array}$ & $\begin{array}{c}0.0232165 \\
(0.0463767)\end{array}$ \\
\hline Constante & $\begin{array}{l}-1.838379 \\
(1.165499)\end{array}$ & $\begin{array}{l}-0.5709393 \\
(0.9288043)\end{array}$ & $\begin{array}{c}-5.399752 * * * \\
(0.764446)\end{array}$ & $\begin{array}{c}-3.680983 * * * \\
(0.9950292)\end{array}$ & $\begin{array}{l}-0.9025195 \\
(0.9067492)\end{array}$ & $\begin{array}{c}-0.8766758 \\
(1.058569)\end{array}$ \\
\hline Municípios - $\mu_{i}$ & Não & Sim & Sim & Sim & Sim & Sim \\
\hline Anos $-\vartheta_{i}$ & Não & Sim & Não & Não & Sim & Sim \\
\hline $\begin{array}{c}\text { Teste de Hausman } \\
\chi^{2} \\
\text { Prob }>\chi^{2}\end{array}$ & & & \multicolumn{2}{|c|}{$\begin{array}{c}19.71 \\
0.0002 \\
\end{array}$} & \multicolumn{2}{|c|}{$\begin{array}{c}32.64 \\
0.0000\end{array}$} \\
\hline $\begin{array}{c}\text { Teste de Chow } \\
\text { Prob }>F\end{array}$ & & & $\begin{array}{c}10.46 \\
0.0000 \\
\end{array}$ & & & \\
\hline $\begin{array}{c}\text { Breusch e Pagan } \\
\text { Prob }>\chi^{2}\end{array}$ & & & & $\begin{array}{l}114.90 \\
0.0000\end{array}$ & & $\begin{array}{l}116.86 \\
0.0000\end{array}$ \\
\hline $\begin{array}{l}\text { Wald } \chi^{2} \\
\text { Prob }>\chi^{2}\end{array}$ & & & & $\begin{array}{l}662.80 \\
0.0000 \\
\end{array}$ & & $\begin{array}{l}734.48 \\
0.0000 \\
\end{array}$ \\
\hline $\begin{array}{c}\text { Teste } F \\
\text { Prob }>F\end{array}$ & $\begin{array}{l}108.81 \\
0.0000\end{array}$ & $\begin{array}{c}30.14 \\
0.0000\end{array}$ & $\begin{array}{l}214.67 \\
0.0000\end{array}$ & & $\begin{array}{l}212.21 \\
0.0000\end{array}$ & \\
\hline$R^{2}$ & 0.5735 & 0.9694 & 0.7731 & 0.7638 & 0.8094 & 0.7915 \\
\hline
\end{tabular}

Nota: *** significância a 1\%, ** a 5\% e * a 10\%; entre parênteses estão as estimativas de desvio-padrão robusto dos parâmetros.

Fonte: Elaboração própria (2019). 
Por outro lado, a depreciação do modelo estimado não apresentou significância estatística. $\mathrm{O}$ modelo demostra que um aumento de $1 \%$ no capital físico (consumo de energia) e capital humano (taxa de alfabetização) provocam uma variação, respectivamente, de 0,19\% e 0,38\% na renda per capita dos municípios do estado de Pernambuco. As variáveis explanatórias do modelo foram capazes de explicar $80,94 \%$ das variações na produção per capita das economias.

Os resultados são compatíveis com a maioria dos trabalhos realizados para o Brasil, onde o capital humano apresenta um percentual de impacto sobre a renda per capita superior ao do capital físico (Firme e Simão, 2019). Os resultados também são condizentes com as estimações de Mankiw et al. (1992), onde o capital humano apresenta um efeito superior ao do capital físico, assim como possui um poder de explicação de $80 \%$ para as variações na produção per capita das economias. Pontua-se, ainda, que a taxa de alfabetização utilizada como proxie para capital humano foi um dos fatores que mais evoluiu no Estado de Pernambuco, tendo em vista, que a média desse indicador por município em 2000 era de 82,1\%, mas em 2010 passou para 92,8\%, gerando uma evolução de $13,05 \%$.

Nesse contexto, os resultados obtidos nesse trabalho são ainda compatíveis com os dos municípios do estado do Ceará, entre 2000 e 2004, através do modelo de Solow ampliando, onde Irffi et al. (2008) demonstra que o aumento de $1 \%$ no estoque do capital humano expresso pela proxie de conclusão do ensino fundamental e médio dos indivíduos provoca um aumento de $0,44 \%$ na renda per capita. Uma análise semelhante feita para os municípios da Região dos Campos Gerais (PR), entre 2001 e 2009, demonstra que o capital humano representado pela proxie de escolaridade média dos trabalhadores formais dos municípios contribui quando ocorre um aumento de $1 \%$ no capital humano em 0,32\% na renda per capita (Cunha e Nunes, 2016).

Por essa razão, o estoque do capital humano estimado para o estado de Pernambuco é reforçado por inúmeros trabalhos realizados no país. As estimativas com o modelo de Solow ampliado, feitas neste trabalho, são coerentes com outros realizados para os municípios brasileiros, entre eles, o de Firme e Simão (2014) utilizado o modelo de MRW para os municípios de Minas Gerais, entre 1991 e 2000, que descobriu que grande parte dos municípios do estado que apresentam elevado crescimento dependem mais do capital humano do que do capital físico, além disso, quando ocorre um aumento de $1 \%$ nessas variáveis a renda per capita varia em $0,40 \%$ e $0,27 \%$.

Esses resultados são condizentes com o modelo de efeito fixo estimado com tendência neste trabalho para os municípios de Pernambuco, entre 2000 e 2010, que são apresentados na Tabela 2, onde podem ser analisados o impacto de cada fator de produção por modelo sobre a renda per capita. Em suma, um aumento de $1 \%$ na taxa de alfabetização impacta entre $0,38 \%$ e 1,41\% nas economias do estado de Pernambuco, a depender do método de estimação realizado, sempre variando os coeficientes em significância estatística entre $1 \%$ e $10 \%$.

Nesse contexto, pontua-se, ainda, que a maioria dos trabalhos que buscam evidenciar o impacto da educação (capital humano) sobre a produção per capita utilizam como proxie a matricula escolar (Barro, 1991) ou anos de escolaridade dos indivíduos, a citar Solow (1956), Lucas (1988), Mankiw et al. (1992), Silva \& Resende (2010) e Cangussu et al. (2010). Portanto, uma contribuição desse trabalho é apresentar a taxa de alfabetização como uma medida possível de ser utilizada para verificar a relação positiva do capital humano com o produto agregado per capita. Além disso, constatou-se que a taxa de alfabetização, dado sua dispersão, apresenta-se como um forte indicador do progresso da economia pernambucana na última década, em virtude da sua grande evolução nesse período.

Este trabalho, também, reforça os importantes achados de Firme e Freguglia (2013), que ao aplicarem o modelo de MRW com dados agrupados em painel para os municípios brasileiros, entre 1980 e 2010, mostraram que o impacto do capital humano é superior ao do capital físico, e que o efeito transbordamento do primeiro fator é mais importante do que o estoque de capital físico. Essa evidência é reforçada por Romer (1990), que aponta a influência do capital humano como motor do progresso tecnológico, promovendo, assim, efeitos diretos e indiretos sobre o crescimento econômico. 
Portanto, conclui-se que os resultados apresentados estão em consonância e harmonia com uma gama de trabalhos sobre o crescimento econômico, contudo, ressalta-se que a proxie utilizada para capital humano (taxa de alfabetização) captou fortemente o efeito da educação sobre o progresso das economias do estado de Pernambuco, apresentando-se com uma excelente variável para estudar os efeitos do capital humano como motor do aumento da renda per capita.

\section{Considerações Finais}

Este estudo atendeu o objetivo proposto, que foi o de investigar o efeito do estoque de capital humano sobre o crescimento da renda per capita dos municípios pernambucanos na primeira década do século XXI. Especificamente, constatou-se que o crescimento da taxa de alfabetização da população pernambucana tem efeito positivo sobre renda per capita, através do modelo MRW.

Nesse sentindo, a principal contribuição desse estudo para a literatura econômica ocorre ao dar ênfase ao papel da alfabetização da população como propulsora do aumento da produção agregada per capita dos municípios do Estado de Pernambuco.

Em geral, os principais resultados obtidos corroboram com a teoria do capital humano e estão em consonância com uma vasta gama de estudos sobre a influência da escolarização da força de trabalho no crescimento econômico, que provam uma correlação positiva entre a elevação no estoque de capacitação e conhecimento dos indivíduos e o aumento do produto de uma economia.

Nesse contexto, após realizados os testes necessários à estimação de um painel de dados balanceado, concluiu-se que o melhor estimador foi de efeito fixo com tendência, ou seja, utilizando-se dummies de tempo. A partir disso, encontrou-se que os parâmetros tanto do capital físico quanto do capital humano são estatisticamente significantes a $1 \%$ e 10\%. Os resultados demonstram que o aumento de $10 \%$ no investimento em capital físico (consumo de energia) e capital humano (taxa de alfabetização) elevam a renda per capita da economia em 1,9\% e 3,8\%, respectivamente.

Deste modo, os resultados obtidos por este trabalho explanam fortemente os efeitos significativos da taxa de alfabetização da população sobre a renda per capita dos municípios do Estado de Pernambuco. Essas estimações reforçam as contribuições de Lucas (1988), Romer (1990) e Mankiw et al. (1992), que discorrem que os investimentos em capital humano sobre o nível de renda de uma economia são superiores aqueles proporcionados pelos investimentos em capital físico.

\section{Referências}

Abramovitz, M. (1986). Catching up, forging ahead, and falling behind. The Journal of Economic History, 46(2), 385-406.

Acevedo, M. C., Montes, I., Maya, J. J. V., González, M. N. V., \& Mejía, T. B. (2007). Capital humano: una mirada desde la educación y la experiencia laboral. Cuadernos de investigación, 56, 1-40.

Atlas de Desenvolvimento Humano no Brasil (2012). Perfil: Educação. Recuperado em 01 de outubro, 2019 de http://twixar.me/jPM1.

Barro, R. J. (1991). Economic growth in a cross section of countries. The quarterly journal of economics, 106(2), 407-443.

Becker, G. S. (1964). Human capital theory. Columbia, New York.

Becker, G. S., Murphy, K. M., \& Tamura, R. (1990). Human capital, fertility, and economic growth. Journal of political economy, 98(5, Part 2), S12-S37. 
Cangussu, R. C., Salvato, M. A., \& Nakabashi, L. (2010). Uma análise do capital humano sobre o nível de renda dos estados brasileiros: MRW versus Mincer. Estudos Econômicos (São Paulo), 40(1), 153-183.

Cunha, A. F., \& Nunes, S. F. (2016). Educação e Crescimento Econômico: Análise dos Municípios da Região dos Campos Gerais do Paraná. Revista de Ensino, Educação e Ciências Humanas, 17(3), 232-239.

Firme, V. D. A. C., \& Simão Filho, J. (2014). Análise do crescimento econômico dos municípios de minas gerais via modelo MRW (1992) com capital humano, condições de saúde e fatores espaciais, 1991-2000. Economia Aplicada, 18(4), 679-716.

Firme, V.D.A.C., \& Simão Filho, J. (2014). Análise do Crescimento Econômico dos Municípios de Minas Gerais através do Modelo de Solow Ampliado, com Inserção de Capital Humano e Condições de Saúde, 1991-2000: Um Estudo de Caso para Econometria Espacial. Área 1 Economia Regional. BNB. Recuperado em 01 outubro, 2019, de http://twixar.me/jgM1.

Firme, V. D. A. C., \& Freguglia, R. S. (2013). Análise do crescimento dos municípios brasileiros utilizando dados em painel e controles espaciais sobre o modelo de mankiw, romer e weil (1992) para o período de 1980 a 2010, in '. XVIII ANPEC Nordeste.

Instituto Brasileiro de Geografia e Estatística [IBGE] (2011). Produto Interno Bruto (PIB) dos Municípios. Recuperado em 01 outubro, 2019, de https://sidra.ibge.gov.br/pesquisa/pibmunic/tabelas.

Irffi, G., Neto, N. T., Oliveira, J. L., Nogueira, C. A. G., Barbosa, M. P., \& Holanda, M. C. (2008). Determinantes do crescimento econômico dos municípios cearenses, uma análise dos dados em painel. Economia do Ceará em debate 2008.

Lucas Jr, R. E. (1988). On the mechanics of economic development. Journal of monetary economics, 22(1), 3-42.

Mankiw, N. G., Romer, D., \& Weil, D. N. (1992). A contribution to the empirics of economic growth. The quarterly journal of economics, 107(2), 407-437.

Mincer, J. (1958). Investment in human capital and personal income distribution. Journal of political economy, 66(4), 281-302.

Nakabashi, L., \& Figueirêdo, L. (2005). Capital humano e crescimento: impactos diretos e indiretos. Textos para discussão CEDEPLAR UFMG, (267).

Secretaria de Desenvolvimento Econômico de Pernambuco (2019). Banco de Dados do Estado (BDE) de Pernambuco. Recuperado em 01 outubro, 2019, www.bde.pe.gov.br.

Romer, P. M. (1990). Endogenous technological change. Journal of political Economy, 98(5, Part 2), S71-S102.

Salgueiro, A. D.S (2012). O papel do capital humano, spillovers e difusão tecnológica no crescimento. Dissertação de mestrado, Universidade Federal do Paraná (UFPA), Curitiba, PR, 2012.

Schultz, T. W. (1961). Investment in human capital. The American economic review, 1-17.

Silva, A. M., \& Resende, G. M. (2010). crescimento econômico comparado dos municípios alagoanos e mineiros: uma análise espacial. Revista Economia Política do Desenvolvimento, 1(6), 135.

Secretaria do Tesouro Nacional [STN] (2011). Sistema de Informações Contábeis e Fiscais do Setor Público Brasileiro (SINCOFI). Recuperado em 01 outubro, 2019, de https://siconfi.tesouro.gov.br/siconfi/pages/public/consulta_finbra/finbra_list.jsf. 
Solow, R. M. (1956). A contribution to the theory of economic growth. The quarterly journal of economics, 70(1), 65-94.

Solow, R. M. (1957). Technical change and the aggregate production function. The review of Economics and Statistics, 312-320.

\title{
Human Capital and Economic Growth: An analysis of the city of Pernambuco between 2000-2010
}

\begin{abstract}
Education is presented as a prime factor for economic growth and development. In this context, this study measured the contribution of the literacy rate on the per capita income growth of the municipalities of Pernambuco, between 2000 and 2010, through the theoretical framework of Solow (1956) and Mankiw et al. (1992). To achieve this goal, a balanced data panel was used, with variables extracted from official sources, citing IBGE, BDE and IPEADATA. The results showed that the $1 \%$ increase in energy consumption (physical capital) and literacy rate (human capital) caused a positive variation of $0.19 \%$ and $0.38 \%$ in per capita income of the municipalities of Pernambuco State. It was also found that in all estimated models the effects of investment in human capital are greater than those of physical capital on per capita income.
\end{abstract}

Keywords: Economic Growth, Human Capital, Education, Pernambuco.

\section{Sobre os Autores}

\section{Álvaro Robério de Souza SÁ}

Graduando em Ciências Econômicas pela Universidade Federal Rural de Pernambuco (UFRPE), Unidade Acadêmica de Serra Talhada (UAST).

Av. Gregório Ferraz Nogueira, S/N, José Tomé de Souza Ramos, Serra Talhada, PE, Brasil, CEP 56909535

E-mail: alvaro.roberiosa@gmail.com

\section{Dhiego Lúcio da SILVA}

Graduando em Ciências Econômicas pela Universidade Federal Rural de Pernambuco (UFRPE), Unidade Acadêmica de Serra Talhada (UAST).

Av. Gregório Ferraz Nogueira, S/N, José Tomé de Souza Ramos, Serra Talhada, PE, Brasil, CEP 56909535

E-mail: dhiego.lucio14@gmail.com

\section{Maria Solange Nunes de Lima SÁ}

Graduada em Administração pela Universidade Federal Rural de Pernambuco (UFRPE), Unidade Acadêmica de Serra Talhada (UAST).

Av. Gregório Ferraz Nogueira, S/N, José Tomé de Souza Ramos, Serra Talhada, PE, Brasil, CEP 56909535

E-mail: marysol.adm@gmail.com 